\title{
Breakdown of integrability in a quasi-one-dimensional ultracold bosonic gas
}

\author{
I.E. Mazets ${ }^{1,2}$, T. Schumm ${ }^{1}$ and J. Schmiedmayer ${ }^{1}$ \\ 1 Atominstitut der Österreichischen Universitäten, TU Wien, A-1020 Vienna, Austria \\ ${ }^{2}$ A.F. Ioffe Physico-Technical Institute, 194021 St. Petersburg, Russia
}

(Dated: November 5, 2018)

\begin{abstract}
We demonstrate that virtual excitations of higher radial modes in an atomic Bose gas in a tightly confining waveguide result in effective three-body collisions that violate integrability in this quasione-dimensional quantum system and give rise to thermalization. The estimated thermalization rates are consistent with recent experimental results in quasi-1D dynamics of ultracold atoms.
\end{abstract}

PACS numbers: 05.30.Jp, 67.85.-d, 03.75.-b

Thermalization does not occur in integrable systems [1], since the number of their integrals of motion equals exactly the number of their degrees of freedom, thus such a system always "remembers" its initial state in the course of its dynamical evolution. In an integrable system the finite spread of initial energy may lead only to relaxation towards the generalized Gibbs (or fully constrained thermodynamic) ensemble [2]. Strictly speaking, there is no thermalization in any closed system, but for non-integrable systems the eigenstate thermalization hypothesis [3] holds, enabling dephasing to mimic the relaxation to the thermal equilibrium.

The Lieb-Liniger model [4] of spinless bosons with contact (point-like) interaction in one-dimension (1D) is a prime example of such an integrable system.

Ultracold atoms in strongly elongated traps with $\omega_{r} \gg$ $\omega_{z}\left(\omega_{r}, \omega_{z}\right.$ being the frequencies of the radial and longitudinal confinement, respectively) are an ideal system for studying $1 \mathrm{D}$ physics as long as both the temperature $T$ and chemical potential $\mu$ are small compared to the energy scale given by the transverse confinement:

$$
\mu<\hbar \omega_{r}, \quad k_{B} T<\hbar \omega_{r} .
$$

Strong inhibition of thermalization was observed in a beautiful experiment with bosons deep in the 1D regime [5]. However, recent experimental results for a weakly interacting Bose gas easily fulfilling the conditions of Eq. (11) [6, 7, 8] are in a good agreement with the thermalequilibrium description of the $1 \mathrm{D}$ atomic ensembles.

In the present letter we investigate the breakdown of integrability and thermalization in ultracold 1D bosons. The key observation is that a radially confined atomic gas is never perfectly $1 \mathrm{D}$, and radial motion can be excited, either in reality or virtually even if Eq. (1) holds. Therefore we call such systems quasi-1D.

We start from identical bosons in a tight waveguide with radial frequency $\omega_{r}\left(\omega_{z}=0\right)$, interacting via the pseudopotential $4 \pi \hbar^{2} m^{-1} \alpha_{s} \delta\left(\mathbf{r}-\mathbf{r}^{\prime}\right)$, where $m$ is the atomic mass, and $\alpha_{s}$ the $s$-wave scattering length:

$$
\hat{\mathcal{H}}_{3 D}=\int d^{3} \mathbf{r}\left[\hat{\psi}^{\dagger}(\mathbf{r})\left(-\frac{\hbar^{2}}{2 m} \frac{\partial^{2}}{\partial z^{2}}+\hat{H}^{(r)}\right) \hat{\psi}(\mathbf{r})+\right.
$$

$$
\begin{gathered}
\left.\frac{2 \pi \hbar^{2} \alpha_{s}}{m} \hat{\psi}^{\dagger}(\mathbf{r}) \hat{\psi}^{\dagger}(\mathbf{r}) \hat{\psi}(\mathbf{r}) \hat{\psi}(\mathbf{r})\right] \\
\hat{H}^{(r)}=-\frac{\hbar^{2}}{2 m}\left(\frac{\partial^{2}}{\partial x^{2}}+\frac{\partial^{2}}{\partial y^{2}}\right)+\frac{m \omega_{r}^{2}}{2}\left(x^{2}+y^{2}\right) .
\end{gathered}
$$

We expand the atomic field operator as follows:

$$
\hat{\psi}(\mathbf{r})=\sum_{n, \ell, k} \hat{a}_{\{n, \ell\} k} \varphi_{n, \ell}(x, y) \frac{\exp (i k z)}{\sqrt{L}} .
$$

Here $L$ is the quantization length and $\varphi_{n, \ell}(x, y)$ is the normalized eigenfunction of both the radial confinement Hamiltonian, $\hat{H}^{(r)} \varphi_{n, \ell}(x, y)=(n+1) \hbar \omega_{r} \varphi_{n, \ell}(x, y)$ and the $z$-projection of the orbital momentum, $-i[x(\partial / \partial y)-$ $y(\partial / \partial x)] \varphi_{n, \ell}(x, y)=\ell \varphi_{n, \ell}(x, y)$. The main quantum number $n=0,1,2, \ldots$, and the orbital-momentum $z$-projection quantum number $\ell$ is restricted by $|\ell|=$ $\bmod (n, 2), \bmod (n, 2)+2, \ldots, n-2, n$ and thus has the same parity as the main quantum number. The atomic annihilation and creation operators $\hat{a}_{\{n, \ell\} k}$ and $\hat{a}_{\{n, \ell\} k}^{\dagger}$ obey the standard bosonic commutation rules.

If two colliding atoms are initially in the transverse ground state of the radial confinement (1D system), then their orbital-momentum quantum numbers after collision are restricted to $-\ell$ and $+\ell$.

For two-body collisions to contribute to thermalization, they have to lead to transverse excitations. This rate of population of the radially excited modes by pairwise atomic collisions $\Gamma_{2 b}$, can be estimated for a nondegenerate Bose gas, using Fermi's golden rule. For $k_{B} T<\hbar \omega_{r}$ this rate is

$$
\Gamma_{2 b} \approx \frac{2 \sqrt{2} \hbar n_{1 D} \alpha_{s}^{2}}{m l_{r}^{3}} e^{-\frac{2 \hbar \omega_{r}}{k_{B} T}}=2 \sqrt{2} \omega_{r} \zeta e^{-\frac{2 \hbar \omega_{r}}{k_{B} T}},
$$

where $n_{1 D}$ is the linear density and $l_{r}=\sqrt{\hbar /\left(m \omega_{r}\right)}$ is the size of the transverse ground state. The dimensionless quantity $\zeta=n_{1 D} \alpha_{s}^{2} / l_{r}$ combines two dimensionless parameters $\left(n_{1 D} \alpha_{s} \propto \frac{\mu}{\hbar \omega_{r}}\right.$ and $\left.\alpha_{s} / l_{r}\right)$ which can be seen as characterizing a $1 \mathrm{D}$ system [9].

Eq. (5) has a transparent physical interpretation: $\Gamma_{2 b}$ is related to the $3 \mathrm{D}$ atomic density, which is $\sim n_{1 D} / l_{r}^{2}$, times the $s$-wave scattering cross-section $\sim \alpha_{s}^{2}$, times the 


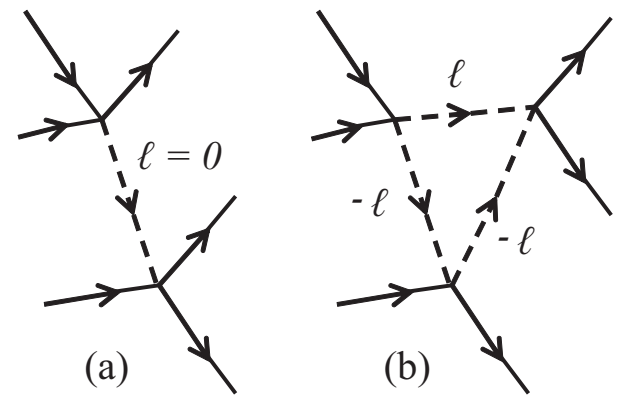

FIG. 1: Feynman diagrams for the effective three-body processes in the second (a) and third (b) orders of perturbation theory. Solid and dashed lines correspond to atoms in the ground and excited states of the radial trapping Hamiltonian, respectively.

exponential Boltzmann factor for the fraction of atoms fast enough to scatter into higher radial modes, times the corresponding velocity of the collision, $\sim \hbar /\left(m l_{r}\right)$.

Calculating the numbers for the data in the ${ }^{87} \mathrm{Rb}$ experiments [7], $\alpha_{s}=5.3 \mathrm{~nm}, n_{1 D}=50 \mu \mathrm{m}^{-1}, \omega_{r} /(2 \pi)=3$ $\mathrm{kHz}, T=30 \mathrm{nK}(\zeta \approx 0.007)$ one obtains a collision rate of $\Gamma_{2 b} \sim 0.02 \mathrm{~s}^{-1}$, at least one order of magnitude too small for the time scale of the experiment.

If the kinetic energy of the collision is less than $2 \hbar \omega_{r}$, then the radial modes can be excited only virtually and contribute to the system dynamics only in the second and higher orders of perturbation theory. If after the collision the radial motion state is $\left|\left\{n_{1}^{\prime}, \ell_{1}^{\prime}\right\},\left\{n_{2}^{\prime}, \ell_{2}^{\prime}\right\}\right\rangle=$ $|\{0,0\},\{2 p, 0\}\rangle$, then only one more collision is enough to quench the virtual excitation and return the system on the energy shell [Fig. 1(a)]. Such a process yields an effective three-body collision already in the second order of perturbation theory.

In contrast processes involving a virtual excitation to $\left|\left\{n_{1}^{\prime},-\ell\right\},\left\{n_{2}^{\prime},+\ell\right\}\right\rangle, \ell \neq 0$, shown in Fig. 1(b), contribute only in the third order, and thus will be neglected.

The small parameter in our perturbation calculation is $n_{1 D} \alpha_{s}$. To avoid complications related to the confinement-induced resonance in $1 \mathrm{D}$ scattering [10] we assume $\alpha_{s} \ll l_{r}[9]$.

In a first step we evaluate the matrix element

$$
\begin{array}{r}
\left\langle\{0,0\},\{2 p, 0\}\left|\delta\left(x-x^{\prime}\right) \delta\left(y-y^{\prime}\right)\right|\{0,0\},\{0,0\}\right\rangle= \\
\left(2^{p+1} \pi l_{r}^{2}\right)^{-1}
\end{array}
$$

that corresponds to two atoms in the ground state of the incoming channel, one atom remaining in the same state, but the other one being excited to a state with zero orbital-momentum quantum number and even main quantum number $n=2 p, p=0,1,2, \ldots(n$ and $\ell$ are required to have the same parity).

The result of Eq. (6) should not be confused with the matrix element where the outgoing channel is characterized by excitation of a higher radial mode of the relative motion of two atoms as discussed in [11], which equals to $\left(2 \pi l_{r}^{2}\right)^{-1}$. The latter is a linear combination of the matrix elements corresponding to vertices on both Fig. 1(a) and Fig. 1(b), and thus can not be applied to the calculation of the second-order process.

Using the matrix element Eq. (6) we can rewrite Eq. (21) and by adiabatically eliminating the radially excited mode operators obtain the effective 1D Hamiltonian:

$$
\begin{aligned}
\hat{\mathcal{H}}_{1 D} & =\hat{\mathcal{H}}_{0}+\hat{\mathcal{H}}_{1 D}^{(3 b)}, \\
\hat{\mathcal{H}}_{1 D}^{(3 b)} & =-\frac{2 \xi \hbar \omega_{r} \alpha_{s}^{2}}{L} \sum \hat{a}_{k_{1}^{\prime}}^{\dagger} \hat{a}_{k_{2}^{\prime}}^{\dagger} \hat{a}_{k_{3}^{\prime}}^{\dagger} \hat{a}_{k_{1}} \hat{a}_{k_{2}} \hat{a}_{k_{3}}
\end{aligned}
$$

where the summation in Eq. (8) is taken over all the kinetic momenta obeying the conservation law $k_{1}^{\prime}+k_{2}^{\prime}+$ $k_{3}^{\prime}=k_{1}+k_{2}+k_{3}, \hat{a}_{k} \equiv \hat{a}_{\{0,0\} k}$ and $\xi=4 \ln (4 / 3) \approx 1.15$. The relative contribution $(\xi-1) / \xi$ of the virtual states with the excitation energy higher than $2 \hbar \omega_{r}$ is remarkably small.

Using Eq. (8) we then obtain the collision rate for the process shown in Fig. 1(a):

$$
\Gamma_{3 b}=C_{3 b} \frac{\hbar n_{1 D}^{2}}{m}\left(\frac{\alpha_{s}}{l_{r}}\right)^{4}=C_{3 b} \omega_{r} \zeta^{2}
$$

with $C_{3 b}=\frac{72 \xi^{2}}{\sqrt{3} \pi^{2}} \approx 5.57$. Comparison to the two-body rates for typical experiments are given in Fig. 2 .

The result of Eq. (9) may seem counterintuitive at first: the collision rate is independent of temperature, and is proportional to $\zeta^{2}$ and the radial confinement $\omega_{r}$.

The physics behind the first observation is related to the fact that the collision kinetic energy is small compared to the virtual excitation energy (according to assumption Eq. (10). Consequently the composite matrix element of the second-order process should not depend (in leading order) on the velocities of colliding particles and hence on temperature (see Eq. (6) ). In addition the phase space volume for the scattered particles is independent on the incoming momenta $k_{1}, k_{2}$, and $k_{3}$.

Since effective three-body elastic scattering is the dominant process the scattering rate must be proportional to the 3D density squared, $\left(n_{1 D} / w_{r}^{2}\right)^{2}$. On the other hand, the scattering rate contains the square of the matrix element corresponding to the diagram in Fig. 1(a), where each vertex is proportional to $\alpha_{s}$, therefore this rate is proportional to $\alpha_{s}^{4}$. The factor $\hbar / m$ provides the correct dimensionality $\left(\mathrm{s}^{-1}\right)$.

We can now compare the scattering rates for the two routes for thermalization and breakdown of integrability in 1D systems: thermally excited two-body collisions $\Gamma_{2 b}$ (Eq. 55) or effective three-body collisions $\Gamma_{3 b}$ (Eq. 9). For $k_{B} T<\hbar \omega_{r}$ we find a simple scaling:

$$
\frac{\Gamma_{3 b}}{\Gamma_{2 b}} \approx \frac{36}{\sqrt{6} \pi^{2}} \zeta e^{\frac{2 \hbar \omega_{r}}{k_{B} T}} \approx 1.97 \zeta e^{\frac{2 \hbar \omega_{r}}{k_{B} T}} .
$$

For large $\zeta$ and small temperatures the scattering rate due to virtual excitations dominates, and can violate integrability even when thermalization processes due to 


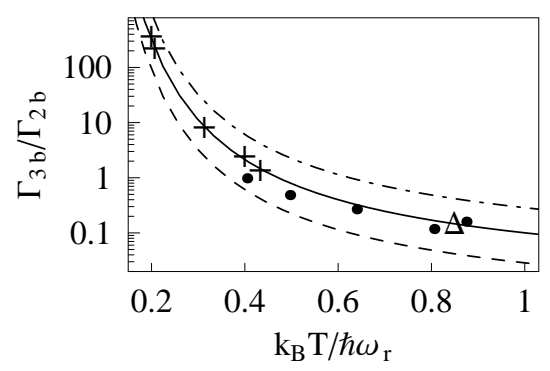

FIG. 2: Ratio between the scattering rates for the two routes to thermalization and breakdown of integrability in 1D systems: $\Gamma_{2 b}$ for two-body collisions leading to excited transverse states, and $\Gamma_{3 b}$ for the effctive three-body collisions. Units on the axes are dimensionless. $\zeta=0.002$ (dashed curve), 0.007 (solid curve), and 0.02 (dot-dashed curve). The points represent the predicted ratios for various sets of experimental parameters from [6] (points), 7] (crosses), and [8] (triangle).

simple two-body collisions are frozen out. A detailed comparison of the two rates and their relation to experimental parameters is given in Fig. 2] The scattering rate due to virtual excitations of the radial modes can dominate over real excitations for typical parameters of the recent experiment [7].

The damping rate of an elementary excitation that moves in a degenerate Bose gas much faster than the speed of sound also has the functional form of Eq. (9), with $C_{3 b}$ being replaced now by $C_{\text {fast }} \approx 13.76$. This result suggests that thermalization is very similar also in the largely unexplored quantum decoherent regime [12] of quasi-1D bosonic dynamics.

To quantify the thermalization, and thereby the violation of integrability in a quasi-1D system by the effective interaction (8), we consider a non-degenerate, weakly-interacting (the Lieb-Liniger parameter [4] $\gamma=$ $2 \alpha_{s} /\left(n_{1 D} l_{r}^{2}\right)$ being much less than 1$)$ gas of bosonic atoms [13] and write out the Boltzmann equation with a threebody collision integral [14], taking into account the indistinguishability of the particles:

$$
\begin{aligned}
\frac{d}{d t} f_{k}= & \frac{72 \xi^{2} \omega_{r}^{2} \alpha_{s}^{4} m}{\sqrt{3} \pi^{3} \hbar} \int_{-K}^{K} \frac{d q}{\sqrt{K^{2}-q^{2}}} \int_{-\infty}^{\infty} d k^{\prime} \int_{-\infty}^{\infty} d k^{\prime \prime} \times \\
& \left(f_{k_{0}-q} f_{k_{+}} f_{k_{-}}-f_{k} f_{k^{\prime}} f_{k^{\prime \prime}}\right) \\
k_{0}= & \frac{k+k^{\prime}+k^{\prime \prime}}{3}, \quad k_{ \pm}=k_{0}+\frac{q}{2} \pm \frac{\sqrt{3}}{2} \sqrt{K^{2}-q^{2}} \\
K= & \frac{2}{3} \sqrt{k^{2}+k^{\prime 2}+k^{\prime \prime 2}-k k^{\prime}-k k^{\prime \prime}-k^{\prime} k^{\prime \prime}}
\end{aligned}
$$

To solve Eq. (11) we use the following ansatz for the perturbed momentum distribution

$$
f_{k}(t)=\frac{n_{1 D}}{\sqrt{\pi} k_{t h}} \exp \left(-k^{2} / k_{t h}^{2}\right)\left[1+\varepsilon_{4}(t) H_{4}\left(k / k_{t h}\right)\right]
$$

where $k_{t h}=\sqrt{2 m k_{B} T} / \hbar$ and $H_{4}$ is the Hermite polynomial of the 4 th order. We choose this form, since

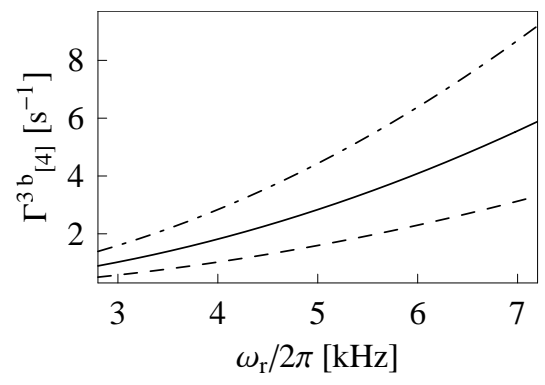

FIG. 3: Dependence of the rate $\Gamma_{[4]}^{3 b}$ of thermalization induced by effective three-body collisions on the radial trapping frequency. $\quad n_{1 D}=30 \mu \mathrm{m}^{-1}$ (dashed curve), $40 \mu \mathrm{m}^{-1}$ (solid curve), and $50 \mu \mathrm{m}^{-1}$ (dot-dashed curve).

it is the simplest nontrivial perturbation that retains $\int d k k f_{k}=0$. Linearizing Eq. (11) with respect to the perturbation amplitude $\varepsilon_{4}(t)$ we obtain the exponential solution $\varepsilon_{4}(t)=\varepsilon_{4}(0) \exp \left(-\Gamma_{[4]}^{3 b} t\right)$ with the decrement

$$
\Gamma_{[4]}^{3 b}=C_{[4]} \frac{\hbar n_{1 D}^{2}}{m}\left(\frac{\alpha_{s}}{l_{r}}\right)^{4}=C_{[4]} \omega_{r} \zeta^{2}
$$

where the numerical constant $C_{[4]}=\frac{64 \xi^{2}}{3 \sqrt{3} \pi^{2}} \approx 1.65$. Taking the perturbation proportional to a higher-order Hermite polynomial $H_{n}$ leads only to a minor the modification of the numerical prefactor, leaving the functional dependence on the parameters of the system unchanged (for example, $n=5$ or 6 increases the thermalization rate by the factor $5 / 4$ or $13 / 9$, respectively). Fig. 3 shows numerical values of $\Gamma_{[4]}^{3 b}$ as a function of the $1 \mathrm{D}$ density of ${ }^{87} \mathrm{Rb}$ atoms and the radial trapping frequency.

Similarly, we calculate numerically the thermalization rate $\Gamma_{[4]}^{2 b}$ for two-body collisions involving the real transitions between the ground and excited radial states. The velocity distribution of atoms in the ground and excited state was perturbed in the same way, as given by Eq. (13), the Boltzmannian distribution of overall populations between the levels being kept intact. In the parameter range of interest we find numerically $\Gamma_{[4]}^{2 b} \approx(0.33 \pm 0.03) \Gamma_{2 b}$, i.e.

$$
\Gamma_{[4]}^{2 b} \approx 0.93 \omega_{r} \zeta e^{-\frac{2 \hbar \omega_{r}}{k_{B} T}} .
$$

The ratio of the thermalization rates for the two-body and three-body processes is very close to the respective ratio of the collision rates, shown in Fig. 2 ,

It is interesting to note that we find for both processes, the two-body collisions to real transverse states and the effective three-body processes via virtual excited states, that thermalization in 1D needs about 3 collisions which are able to distribute energy. This is very close to the 2.7 collisions required for thermalization in $3 \mathrm{D}$ 15].

For the typical parameters of an ultracold ${ }^{87} \mathrm{Rb}$ gas on an atom chip [7] $\left(\omega_{r} \approx 2 \pi \times 3 \mathrm{kHz}, n_{1 D} \approx 50 \mu \mathrm{m}^{-1}\right)$ 
we obtain $\Gamma_{[4]}^{3 b} \approx 2 \mathrm{~s}^{-1}$. This thermalization rate is temperature-independent and much larger than the one calculated from the simple two-body collisions with the energy sufficient to excite radial modes $\Gamma_{[4]}^{2 b} \approx 3 \times$ $10^{-3} \mathrm{~s}^{-1}$ at the lowest temperatures measured (30 nK). The estimated $\Gamma_{[4]}^{3 b}$ is consistant with the time needed for evaporative cooling of a ${ }^{87} \mathrm{Rb}$ gas on an atom chip well below $\hbar \omega_{r}$ [6, 7].

Comparison of our thermalization rates to the experimental results of Kinoshita, Wenger and Weiss [5] is more qualitative. In this experiment, a degenerate ${ }^{87} \mathrm{Rb}$ gas in a two-dimensional optical lattice $\left(\omega_{r} \approx 2 \pi \times 67 \mathrm{kHz}\right.$, $\left.n_{1 D} \approx 10 \mu \mathrm{m}^{-1}\right)$ is split by a laser Bragg pulse into two groups with opposite kinetic momenta, which begin to oscillate in a weak trapping potential in $z$-direction, colliding each half-period of the oscillation. The experiment is close to the strongly interacting regime $(\gamma \sim 1)$, and one has to take the strong suppression of three-body collisions into account. The rate of relaxation of the relative motion of the two groups of atoms can be written as

$$
\Gamma_{r m}=C_{r m} \eta_{\tau} \omega_{r} \zeta^{2} \mathcal{F}(\gamma)
$$

where $C_{r m} \sim C_{3 b}$ and $\eta_{\tau}<1$ is the fraction of the period, when the two groups of atoms with opposite velocities overlap in space. The dominant factor in Eq. (16) is the suppression of three-body scattering $\mathcal{F}(\gamma)$ via atomatom correlations in both initial and final states. The experiment [16] showed 7-fold suppression of three-body inelastic losses in a quasi-1D bosonic gas with $\gamma \approx 0.5$ compared to a regular BEC. However, there is a difference between elastic scattering discussed here and that of Ref. [16] where the final state corresponds to a molecule and the fast atom carrying away the energy and momentum released in the three-body recombination process. In the elastic scattering related to dissipation the suppression enters in both the incoming and the outgoing channel and should be the square of the measured value. Reading the predictions for the parameters in $[5](\gamma \approx 1.4)$ from the estimates in [16] we find a suppression factor $\mathcal{F}(1.4) \sim 4 \cdot 10^{-4}$ and $\Gamma_{r m} \lesssim 0.03 \mathrm{~s}^{-1}$, which is consistent with the experimentally obtained lower bound to the relative motion damping rate $\Gamma_{r m}^{-1}>20 \mathrm{~s}$.

The mechanism discussed here which leads to the breakdown of integrability in $1 \mathrm{D}$ is to a certain extent similar to the virtual association of atoms to a molecular dimer [17]. In the present discussion, virtual excitation of radial modes during a two-atom collision temporarily localize the interatomic distance on the length scale $\sim l_{r}$. Scattering a third atom on such a transient structure of finite size and mass $2 m$ leads to thermalization and vio- lates integrability. In Ref. [17], collisions of a third atom bring "virtual" dimers, enhanced in size by a Feshbach resonance down to the energy shell, thus bringing about "quantum chemistry" in 1D.

To summarize, we identify effective three-body collisions which arise in the second order of perturbation theory as a mechanism of breakdown of integrability and, hence, thermalization in $1 \mathrm{D}$ atomic gases. These processes are associated with virtual excitation of radial modes and for weakly interacting quasi-1D Bose gases can be dominant at $k_{B} T<\hbar \omega_{r}$. Our estimations of the relaxation rates in are consistent with recent experimental observations [5, 6, 7].

This work is supported by the EC (STREP MIDAS) the INTAS and the FWF. I.E.M. acknowledges the Lise Meitner Fellowship (FWF).

[1] H.B. Thacker, Rev. Mod. Phys. 53, 253 (1981).

[2] M. Rigol, V. Dunjko, V. Yurovsky, and M. Olshanii, Phys. Rev. Lett. 98, 050405 (2007).

[3] M. Srednicki, Phys. Rev. E 50, 888 (1994); M. Rigol, V. Dunjko, and M. Olshanii, arXiv: cond-mat/07081324.

[4] E.H. Lieb and W. Liniger, Phys. Rev. 130, 1605 (1963); E.H. Lieb, Phys. Rev. 130, 1616 (1963).

[5] T. Kinoshita, T. Wenger, and D.S. Weiss, Nature 440, 900 (2006).

[6] S. Hofferberth et al., Nature 449, 324 (2007).

[7] S. Hofferberth, et al., arXiv: cond-mat/0710.1575.

[8] A.H. van Amerongen, et al., arXiv: cond-mat/0709.1899.

[9] The effective 1D coupling constant of pairwise interacting bosonic atoms in a waveguide changes with confinement as $g_{1 D}=2 \hbar \omega_{r} \alpha_{s} /\left[1-\frac{C^{\prime} \alpha_{s}}{\sqrt{2} l_{r}}\right], C^{\prime} \approx 1.46[10]$ due to virtual excitation of the radial modes and increases as $\alpha_{s} / l_{r}$ grows. This points to the ratio $\alpha_{s} / l_{r}<1$ as the measure how much the 1D approximation is violated.

[10] M. Olshanii, Phys. Rev. Lett. 81, 938 (1998).

[11] V.A. Yurovsky, Phys. Rev. A 71, 012709 (2005).

[12] J.-B. Trebbia, J. Esteve, C.I. Westbrook, and I. Bouchoule, Phys. Rev. Lett. 97, 250403 (2006); I. Bouchoule, K.V. Kheruntsyan, and G.V. Shlyapnikov, Phys. Rev. A 75, 031606(R) (2007).

[13] The assumptions of weak interaction and non-degeneracy enable us to express the three-particle distribution function through the product of single-particle distribution functions $f_{k}$. In contrast, calculation of relaxation via three-body collisions of low-energy excited states of the Lieb-Liniger model, especially for $\gamma>1$, requires taking into account strong correlations in the quasi-1D bosonic system.

[14] M.S. Green, Phys. Rev. 136, A905 (1964).

[15] H. Wu and C. J. Foot, J. Phys. B 29, L321 (1996).

[16] B.L. Tolra et al., Phys. Rev. Lett. 92, 190401 (2004).

[17] V.A. Yurovsky, A. Ben-Reuven, and M. Olshanii, Phys. Rev. Lett. 96, 163201 (2006). 